\title{
Early surgical management and long-term surgical outcome for intraventricular hemorrhage-related posthemorrhagic hydrocephalus in shunt-treated premature infants
}

\author{
Hans Christoph Bock, Dr Med, Jacqueline Feldmann, and Hans Christoph Ludwig, Dr Med \\ Pediatric Neurosurgery Section, Department of Neurosurgery, University Medical Center Göttingen, Germany
}

OBJECTIVE Perinatal intraventricular hemorrhage (IVH) in premature neonates may lead to severe neurological disability and lifelong treatment requirement for consecutive posthemorrhagic hydrocephalus (PHHC). Early CSF diversion as a temporizing measure, or a permanent ventriculoperitoneal shunt (VPS), is the treatment of choice. Preterm neonates are not only at high risk for different perinatal but also for treatment-related complications. The authors reviewed their institutional neurosurgical management for preterm neonates with IVH-related PHHC and evaluated shunt-related surgical outcome for this particular hydrocephalus etiology after completion of a defined follow-up period of 5 years after initial shunt insertion.

METHODS The authors retrospectively analyzed early surgical management for preterm newborns who presented with IVH and PHHC between 1995 and 2015. According to the guidelines, patients received implantation of a ventricular access device (VAD) for temporizing measures or direct VPS insertion as first-line surgical treatment. Surgical outcome was evaluated for a subgroup of 72 patients regarding time to first shunt revision and the mean number of shunt revisions during a time span of 5 years after initial shunt insertion. Gestational age (GA), extent of IVH, and timing and modality of initial surgical intervention were analyzed for potential impact on corresponding surgical outcome.

RESULTS A total cohort of 99 preterm newborns with GAs ranging from 22 to 36 weeks (mean 28.3 weeks) with perinatal IVH-related PHHC and a median follow-up duration of 9.9 years postpartum could be selected for further investigation. Extent of perinatal IVH was defined as grade III or as periventricular hemorrhagic infarction in $75 \%$ of the patient cohort. Seventy-six patients (77\%) underwent VAD insertion and temporizing measures as initial surgical treatment; for $72(95 \%)$ of these a later conversion to permanent ventriculoperitoneal shunting was performed, and 23 patients received direct VPS insertion. Etiological and treatment-related variables revealed no significant impact on revision-free shunt survival but increased the mean numbers of shunt revisions after 5 years for low GA, higher-order IVH in the long term.

CONCLUSIONS Low GA and higher-order IVH in preterm neonates with PHHC who are treated with VPSs show no significant impact on time to first shunt revision (i.e., revision-free shunt survival), but marked differences in mean revision rates evaluated after completion of 5 years of follow-up. Temporizing measures via a VAD represent a rational strategy to gain time and decision guidance in preterm patients with PHHC before permanent VPS insertion.

https://thejns.org/doi/abs/10.3171/2018.1.PEDS17537

KEYWORDS posthemorrhagic hydrocephalus; preterm hemorrhage; shunt; outcome

$\mathrm{P}$ ERINATAL intraventricular hemorrhage (IVH) initiating from the substantial (but immature) vascularized germinal matrix is a major complication of prematurity and may lead to lifelong consequences like cerebral palsy, developmental disorders, and mental defi- ciencies. ${ }^{3,19}$ A subset of infants with perinatal IVH develops posthemorrhagic ventricular dilation (PHVD) and/or progressive posthemorrhagic hydrocephalus (PHHC). To date no evidence-based guidelines exist to define optimal management for preterm neonates who develop IVH-relat-

ABBREVIATIONS DPV = differential pressure valve; $\mathrm{GA}=$ gestational age; $\mathrm{HR}=$ hazard ratio; $\mathrm{IVH}=$ intraventricular hemorrhage; $\mathrm{PHHC}=$ posthemorrhagic hydrocephalus; PHVD = posthemorrhagic ventricular dilation; PVHI = periventricular hemorrhagic infarction; RFSS = revision-free shunt survival; VAD = ventricular access device; VPS = ventriculoperitoneal shunt; VSG = ventriculosubgaleal.

SUBMITTED October 16, 2017. ACCEPTED January 30, 2018.

INCLUDE WHEN CITING Published online May 4, 2018; DOI: 10.3171/2018.1.PEDS17537. 
ed PHHC concerning indications, modalities, and timing of surgical treatment. ${ }^{15}$ A European survey determined considerable variation in the diagnostic and therapeutic approaches regarding neonates with PHVD. ${ }^{6}$

Pediatric patients requiring permanent CSF diversion by ventriculoperitoneal shunting at a young age are suspected to be at higher risk for repeated shunt complications and failure. . $, 8,22,24$ Hence, for premature infants with PHVD after IVH, different options for surgical temporizing measures have been described to drain CSF and at the same time estimate clinical progression potentially resulting in PHHC. Serial lumbar punctures, ventriculosubgaleal (VSG) shunts, external ventricular drainage, and ventricular access devices (VADs) are well-described interventions. ${ }^{2,11,13}$ Evidence could be demonstrated for VADs to reduce morbidity and mortality compared with external ventricular drainage. Furthermore, VSG shunts reduce the need for daily CSF aspiration and consequently the infection risk compared with VADs..$^{10,13}$

For the most common techniques used to temporize PHVD, no significant difference could be found when comparing VSG shunts and VAD. ${ }^{2}$ A current prospective cohort study found no evidence for impact on the conversion rate to permanent ventriculoperitoneal shunting related to the choice of temporizing technique. ${ }^{24}$ The multiplicity of causes of pediatric hydrocephalus with the variety of associated diseases, complications of treatment, and the inherent complexity of the patient population make reliable data on outcomes difficult to obtain. ${ }^{22}$ In regard to outcome in adults with hydrocephalus suffered during childhood, shunt treatment has demonstrated a substantial effect on social participation in later life. ${ }^{16}$ A systematic literature review aiming to provide evidence-based guidelines for various issues concerning the management of pediatric hydrocephalus illustrated the lack of convincing data especially in regard to PHHC.,, 15 To review our established institutional neurosurgical management practices for preterm neonates with perinatal IVH and PHHC, we analyzed our institution's pediatric hydrocephalus and shunt registry ${ }^{5}$ for ventriculoperitoneal shunt (VPS)-related surgical outcome in preterm patients with PHHC.

\section{Methods}

By using our daily updated institutional database comprising the complete surgical history of pediatric patients with hydrocephalus back to 1995 , we analyzed the clinical course of all patients conforming to the following inclusion criteria: prematurity ( $<37$ weeks of gestation), ultrasoundverified perinatal IVH accompanied by PHVD, complete institutional surgical history, and follow-up interval of at least 12 months. Grading of IVH referred to the maximum extent of IVH on any side that was detectable on transcranial ultrasound (Volpe grade). ${ }^{23}$ Patients with any hydrocephalus-related neurosurgical intervention received at another institution, gestational age (GA) $\geq 37$ weeks, ventricular dilation related to any other cause than perinatal IVH, or missing corresponding follow-up data were excluded from further investigation.

Our institutional pathway for the management of IVHrelated PHVD, established in 1995 and still followed since then, determines primary temporizing measures in terms of VAD insertion and a CSF-aspiration regimen for newborns with a body weight below $2500 \mathrm{~g}$ or in restricted clinical condition. Insertion of VPSs in patients requiring permanent CSF diversion presumed a body weight of at least $2500 \mathrm{~g}$, sufficient clinical condition, and no signs of infection. The VADs were placed via a small craniotomy, usually into the left ventricular frontal horn, for repeated CSF aspirations of 2-5 $\mathrm{ml}$ up to 3 times per day, depending on daily clinical examination and cranial ultrasound findings. In case of increasing ventricular dilation, accelerating head circumference, other clinical signs of raised intracranial pressure, or failing gradual reduction of CSF aspiration, VAD revision and conversion to contralateral ventriculoperitoneal shunting was performed under the conditions mentioned above. To secure data reliability, only patients who completed a defined follow-up of 5 years $(n=72)$ were considered for shunt-related surgical outcome evaluation. The mean number of cumulative shunt revisions within 5 years after initial VPS insertion was analyzed with regard to GA ( $<28$ weeks vs 28-36 weeks), maximum extent of IVH (Volpe grade I-II vs grade III or periventricular hemorrhagic infarction [PVHI]), sex, postnatal age at initial VPS insertion ( $<3$ months vs $\geq 3$ months), initial surgical management (VAD before shunting vs direct VPS insertion), and valve design for VPSs (fixed differential pressure valve [DPV] vs adjustable DPV). Additionally, Kaplan-Meier analysis with log-rank test, hazard ratio (HR), and $95 \%$ CI was used to investigate the potential impact of any of these variables on revision-free shunt survival (RFSS). The study was approved by the ethical review committee of the University Medical Center Göttingen.

\section{Results}

\section{Preterm Patients With IVH}

Out of the total of 461 pediatric patients with hydrocephalus of different etiology registered in our institutional database between 1995 and the time of evaluation, clinical data in 99 preterm neonates ( 34 female, 65 male) with IVH and PHVD who met the inclusion criteria mentioned above could be separated for further evaluation. Twentythree preterm patients with incomplete follow-up, any hydrocephalus-related surgery at another institution, or hydrocephalus etiology different from IVH were excluded during the data selection process. The cohort of 99 preterm patients with PHHC (Table 1) presented with a mean birth weight of $1227 \mathrm{~g}$ (range 535-2340 g) and a mean GA of 27 weeks (range 22-36 weeks) at birth. Fifteen patients (15\%) were born between weeks 32 and 36; 36 (36\%) between weeks 28 and 31; and 48 patients (49\%) before the 28th week of gestation. Initial IVH was assessed by cranial ultrasound examination in the pediatric ICU and defined as Volpe grade I-II in $25 \%$, and grade III or PVHI in $37.5 \%$ each of the patient cohort. Distribution of IVH grading in correlation to GA revealed the highest percentage of PVHI $(46 \%)$ in preterm patients born earlier than 28 weeks of gestation (Fig. 1A). In 50\% of the cohort, initial neurosurgical intervention was necessary within the first 30 days postpartum (Fig. 1B). Patients underwent initial VAD insertion and temporizing measures in 76 cases (77\%), and 
TABLE 1. Characteristics in all 99 preterm neonates with IVH-related PHHC

\begin{tabular}{cc}
\hline Characteristic & No. of Patients \\
\hline GA at birth & \\
\hline$<28$ wks & 48 \\
\hline $28-31$ wks & 36 \\
\hline $32-36$ wks & 15 \\
\hline Grading of IVH* & 25 \\
\hline Grade I-II & 37 \\
\hline Grade III & 37 \\
\hline PVHI & 65 \\
\hline Sex & 34 \\
\hline Male & \\
\hline Female & 23 \\
\hline Surgical management & 72 \\
\hline Direct VPS & 4 \\
\hline VAD before VPS &
\end{tabular}

* According to Volpe's classification.

72 of these received secondary conversion to ventriculoperitoneal shunting. The VAD insertion was performed by consultant neurosurgeons in $96 \%$ of all cases, with a mean operating time of 23 minutes. In 4 cases, no permanent VPS insertion was indicated, because PHVD resolved spontaneously after temporizing measures were used. The VADs were inserted within the first 30 days after birth in $64 \%$, and within the first 3 months postpartum in $92 \%$ of all cases. Revision of primary VADs due to surgical complications was indicated in a total of 10 patients (13\%), local wound healing problems or infections were found in 7 , and occlusion with or without CSF leakage occurred in 3 cases.

\section{Surgical Management of Preterm PHHC}

For 95 of 99 patients with preterm-related IVH, primary $(24 \%)$ or secondary $(76 \%)$ VPS insertion was indicated. The VPS surgery was performed by consultant neurosurgeons in $97 \%$ of cases, with a mean operating time of 53 minutes. Initial VPS treatment without previous VAD insertion was performed in 23 patients, and this surgery was carried out within the first 3 months postpartum in $17 \%$ and within the first 30 days in $4 \%$ (Fig. 1B). For those with secondary VPS implantation, the median time interval between primary VAD insertion and conversion to ventriculoperitoneal shunting was 1.6 months (Fig. 1C). Valve design for initial shunt implantation differed in regard to contemporary availability and represented fixed DPVs in 23 of $95(24 \%)$ and adjustable DPVs in 72 of $95(76 \%)$ of all patients who received VPSs. Reasons for initial shunt failure could be attributed to infection in 8 patients (8\% infection rate) and various mechanical complications. Shunt infections were diagnosed if clinical signs of infection (fever, seizures, lethargy) were correlated with elevated white blood cell count in CSF aspirated from the shunt reservoir, with or without microbiological proof. Malfunctions that induced initial surgical shunt revision were allocated in relation to time of occurrence and defined as early $(<12$ months) or later ( $\geq 12$ months) complications after VPS insertion. The predominant causes for initial shunt failure collectively could be attributed to growth-related catheter migration, occlusion, and shunt infection, in decreasing order. Shunt infections and local wound healing problems were found to occur early, and growth-related catheter migration, overdrainage, and disconnections occurred rather late after initial VPS insertion (Fig. 2B).
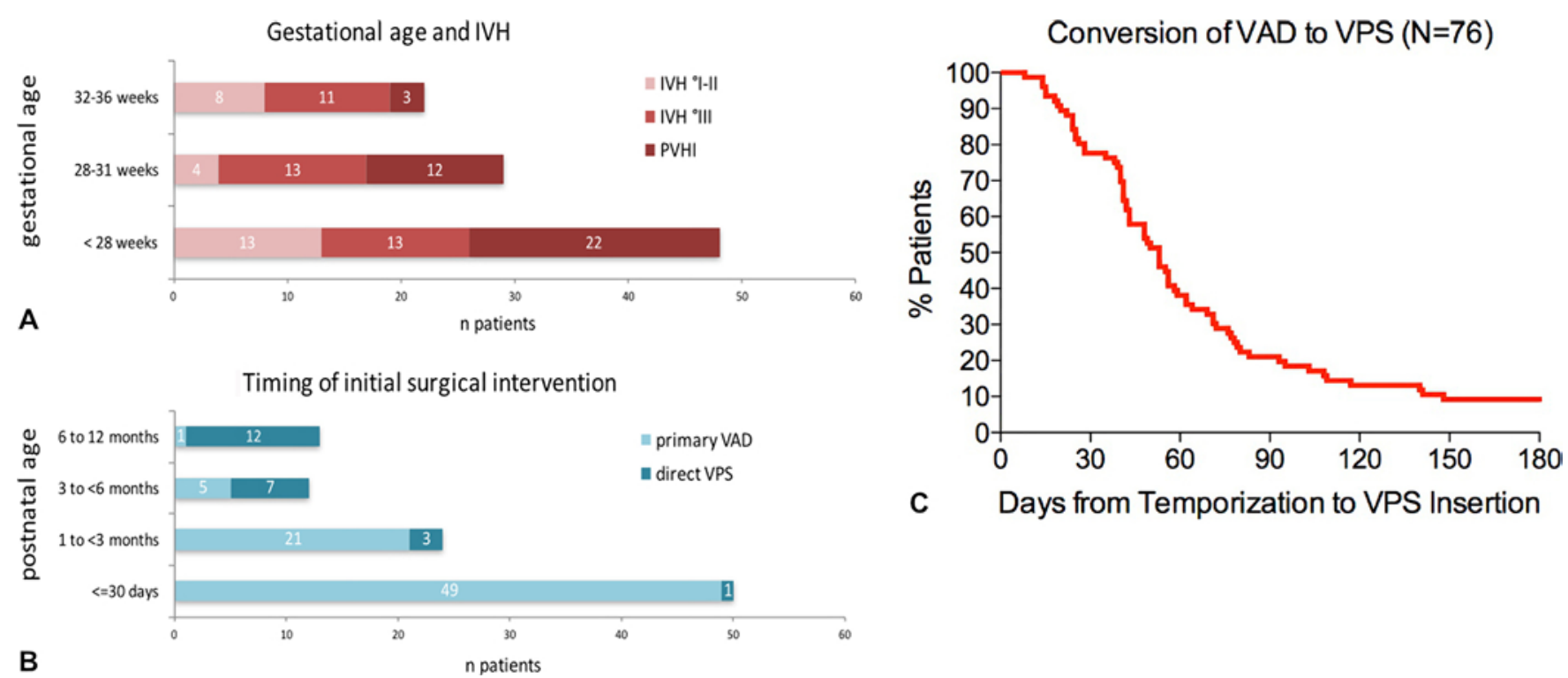

C Days from Temporization to VPS Insertion

FIG. 1. A: Grading of perinatal IVH in correlation to GA for 99 preterm neonates with PHVD. B: Time of initial neurosurgical intervention in terms of VAD insertion with temporizing measures or direct insertion of a permanent VPS. C: Time to conversion of VAD to permanent VPS over the time (days) after VAD insertion. Figure is available in color online only. 


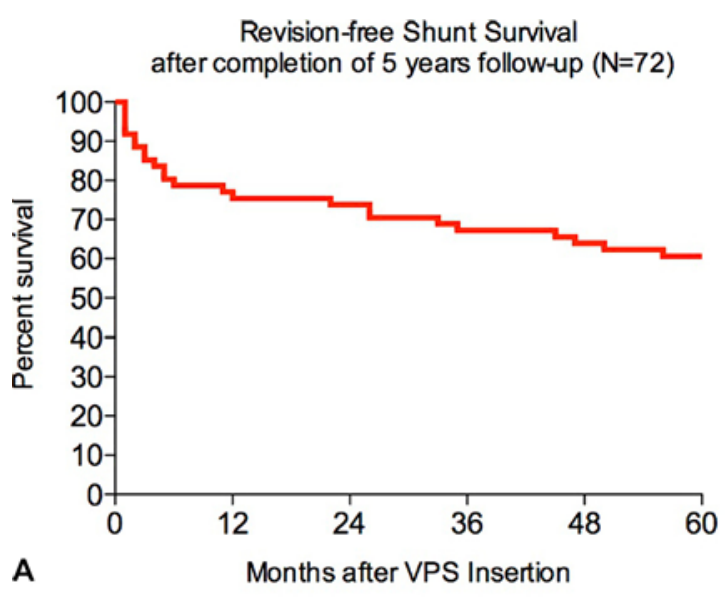

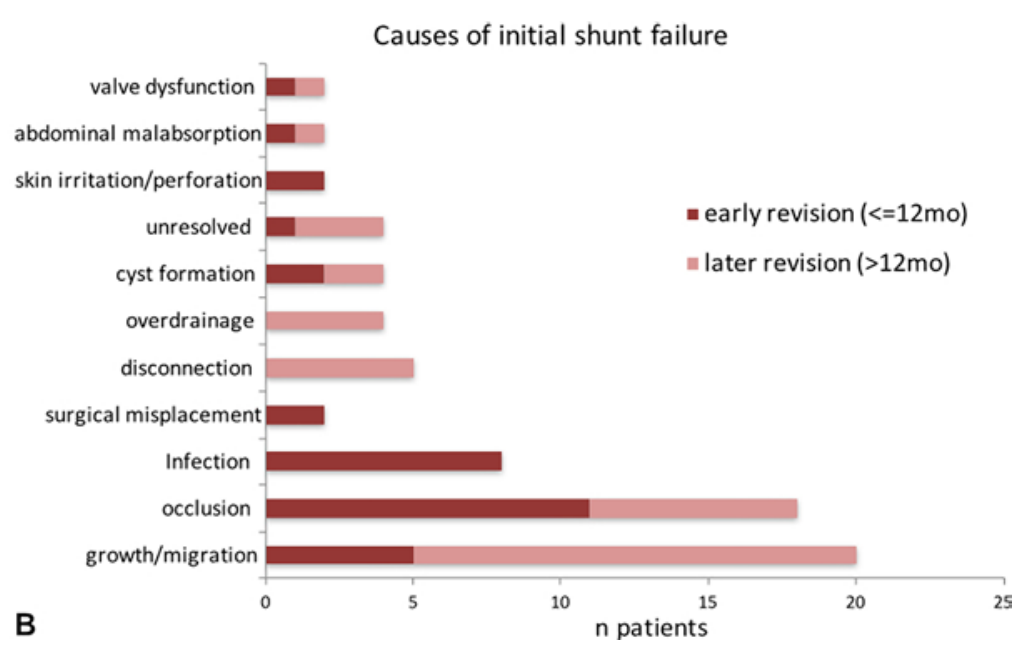

FIG. 2. A: Kaplan-Meier analysis of RFSS for 72 preterm patients with IVH-related PHHC over a defined follow-up of 5 years. B: Causes of initial shunt failure in VPS-treated preterm neonates with PHHC correlated to timewise occurrence within the first 12 months (early revision) or after the passage of 12 months (later revision) following initial VPS placement. Figure is available in color online only.

\section{Surgical Outcome 5 Years After VPS Insertion}

Shunt-related surgical outcome was evaluated for a subgroup of 72 patients who all provided complete followup after a defined interval of 5 years (median 11.8 years) after initial VPS insertion (Table 2). After the 1st year, $35 \%$; after the 3rd year, $43 \%$; and after 5 years of followup, $49 \%$ of all patients received at least one shunt revision (mean 1.0 revisions after 5 years). Preterm neonates born before 28 weeks of gestation $(n=39)$ were found to have an increased mean number of revisions after completion of 5 years of follow-up (mean 1.3 revisions) compared with patients born between weeks 28 and 36 of gestation ( $\mathrm{n}=33$, mean 0.7 revisions). A concordant increase in mean revision rates 5 years after shunt insertion could be found for patients with IVH grade III or PVHI $(n=56$, mean 1.2 revisions) compared with those with IVH grade I-II ( $\mathrm{n}=16$, mean 0.6 revisions). Patients who received VADs and temporization before VPS insertion $(n=53)$ revealed decreased mean revisions (mean 0.9 revisions) after 5 years of follow-up in comparison with patients who received direct VPS insertion ( $\mathrm{n}=19$, mean 1.4 revisions).

TABLE 2. Outcome after completion of 5 years of follow-up in 72 VPS-treated patients with perinatal IVH

\begin{tabular}{|c|c|c|c|c|}
\hline Variable & No. of Patients (\%) & $\%$ of Revised Shunts & Mean Revisions/Patient & Mean RFSS (mos) \\
\hline Totals & $72(100)$ & 49 & 1.0 & 64 \\
\hline \multicolumn{5}{|l|}{ GA at birth } \\
\hline$<28$ wks & $39(54)$ & 54 & 1.3 & 59 \\
\hline $28-36$ wks & $33(46)$ & 42 & 0.7 & 69 \\
\hline \multicolumn{5}{|l|}{ Grading of IVH* } \\
\hline Grade I-II & $16(22)$ & 38 & 0.6 & 67 \\
\hline Grade III or PVHI & $56(78)$ & 52 & 1.2 & 63 \\
\hline \multicolumn{5}{|l|}{ Sex } \\
\hline Male & $51(71)$ & 47 & 1.1 & 64 \\
\hline Female & $21(29)$ & 52 & 1.0 & 63 \\
\hline \multicolumn{5}{|c|}{ Postnatal age at initial VPS insertion } \\
\hline$<3 \mathrm{mos}$ & $27(37)$ & 48 & 0.9 & 64 \\
\hline $3-11 \mathrm{mos}$ & $45(63)$ & 49 & 1.1 & 64 \\
\hline \multicolumn{5}{|l|}{ Surgical management } \\
\hline Direct VPS & $19(26)$ & 47 & 1.4 & 65 \\
\hline VAD before VPS & $53(74)$ & 49 & 0.9 & 64 \\
\hline \multicolumn{5}{|l|}{ Valve design } \\
\hline Fixed DPV & $21(29)$ & 52 & 1.2 & 68 \\
\hline Adjustable DPV & $51(71)$ & 47 & 1.0 & 62 \\
\hline
\end{tabular}

* According to Volpe's classification. 


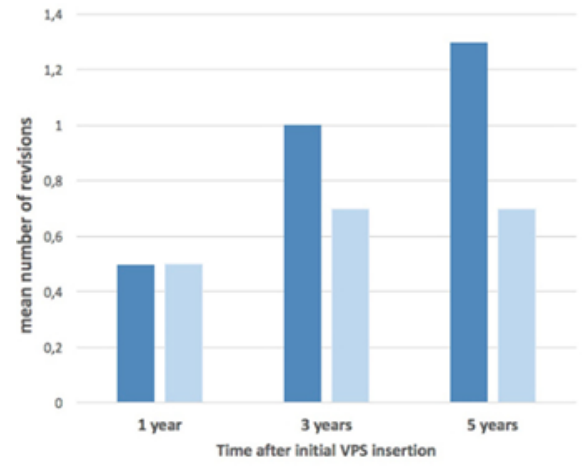

A
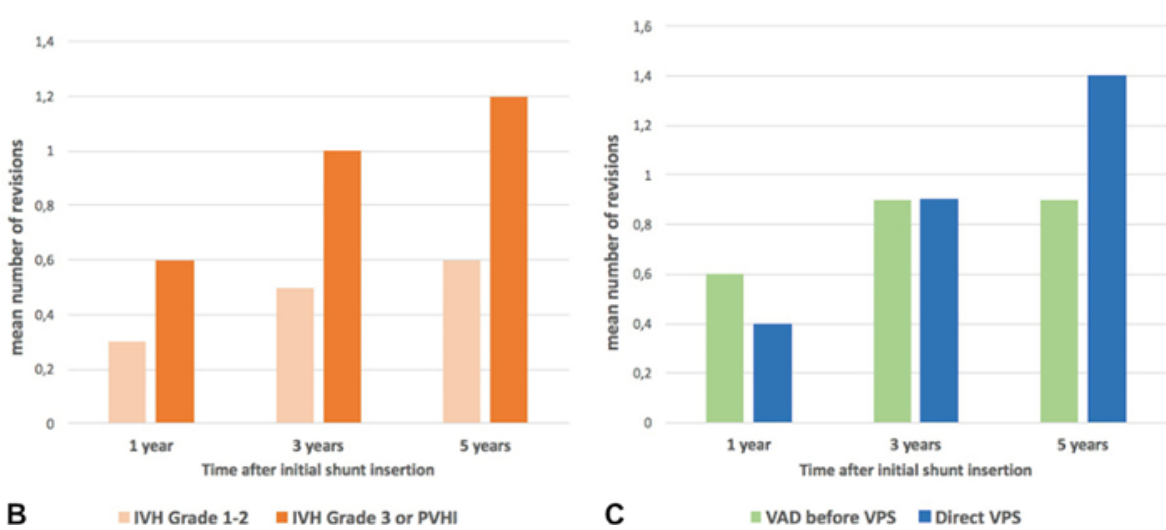

C

FIG. 3. Bar graphs showing the mean cumulative number of shunt revisions at 1, 2, and 5 years after initial shunt insertion, in reference to GA (A), extent of IVH (B), and modality of initial surgical intervention (C). Figure is available in color online only.

The mean number of revisions after the 1st, 3rd, and 5th year after shunt insertion are illustrated in Fig. 3. No significant impact on time to first revision (i.e., RFSS) could be found in reference to GA ( $\mathrm{p}=0.85$, HR $0.925,95 \%$ CI 0.411-2.079); extent of IVH ( $\mathrm{p}=0.59$, HR $0.775,95 \%$ CI 0.307-1.958); postnatal age at initial shunt insertion ( $p$ $=0.571, \mathrm{HR} 1.275,95 \%$ CI $0.549-2.961)$; valve design $(\mathrm{p}$ $=0.175$, HR $0.540,95 \%$ CI $0.221-1.316)$; or modality of initial neurosurgical intervention $(\mathrm{p}=0.978$, HR 0.9876 , 95\% CI 0.404-2.41) within the group of 72 patients after completion of 5 years of follow-up.

\section{Discussion}

\section{Early Surgical Intervention}

Decision making for treatment modality and optimal surgical time frame in prematurity-related PHHC remains difficult. The complex combination of hemorrhage-induced direct CNS damage, space-occupying CSF accumulation in a condition of somatic immaturity, and consequent vulnerability lead to controversial preferences for indication and type of surgical treatment. ${ }^{6}$ Besides the IVH-related injury, respiratory and cardiac disorders as well as general predisposition for infection frequently complicate the early neonatal course. ${ }^{7,19}$ The impact of immaturity in preterm neonates on the one hand may motivate early invasive attempts to reduce the potential risk of raised intracranial pressure or, on the other hand, demand containment to prevent additional surgery-related morbidity.

\section{Initial and Recurrent Shunt Failure}

A prospective multicenter cohort study investigated potential risk factors with an impact on time to initial shunt failure for pediatric patients and described the following variables: 1$)$ young age ( $\leq 6$ months); 2 ) a preexisting cardiac comorbidity; and 3) the intraoperative use of an endoscope as being independently associated with a higher risk for initial shunt failure. ${ }^{18}$ In this earlier study, IVHrelated hydrocephalus in preterm infants was the predominant etiology in the patient cohort (21.8\%), but could not demonstrate independent impact on RFSS itself over a median follow-up of 400 days. Evaluating shunt-related surgical outcome for this exceptionally vulnerable patient subgroup requires thorough and gapless follow-up with regard not only to the time to initial shunt failure but also to the entire group of treatment-associated complications in the long term. Therefore, not only RFSS, but also objective long-term variables like average cumulative shunt revisions were taken into consideration for the surgical outcome evaluation in our study. Within the group of all shunt-treated preterm patients with IVH-related PHHC, we found higher mean revision rates for patients born before 28 weeks of gestation and for those with higher-order IVH. Therefore, immaturity of the affected brain, extent of tissue damage, and amount of disseminated blood may be the key events not only for neurodevelopmental outcome but also for shunt-related surgical outcome in the long term.

\section{Counteracting Shunt Complications}

Insertion of VADs with temporizing measures in premature patients with PHHC aims for stepwise reduction of intraventricular blood and a more mature CNS before finalizing the decision about permanent CSF diversion. This effect might be reflected by our findings of fewer mean revisions for patients initially treated with VADs compared with those who received direct shunt insertion after completion of 5 years of follow-up. Predispositions for early and multiple shunt failure have been reported previously in other series concerning surgical outcome of pediatric PHHC. ${ }^{4,8,24}$ The few studies related to the question of any advancements in surgical outcome for this subgroup from past to present offer controversial statements. A metaanalytical model found no evidence for improvements in shunt failure rates in pediatric patients over the years..$^{21} \mathrm{In}$ contrast, a comparison of the Hydrocephalus Clinical Research Network (HCRN) cohort with historical controls determined improvements for current outcomes of shunting in general pediatric neurosurgery practice, although the reason remained unclear. ${ }^{12}$ Other studies concentrating on neurological outcome after preterm intracranial hemorrhage indicate that long-term motor and cognitive deficiencies should be ascribed to the extent of preceding hemorrhage rather than to any factors appearing after the first surgical CSF diversion. ${ }^{14,17}$

Within the group of preterm patients with IVH-related 
PHHC in our study, etiological and treatment-related variables did not show significant impact on time to first shunt revision (i.e., RFSS). Still, our investigations demonstrate that lower GA ( $<28$ weeks) and higher-order IVH (Volpe grade III and PVHI) were correlated with slightly lower mean RFSS than average within this particular group of patients. The complex comorbidity of prematurity itself, and the additional pathology of a cerebral hemorrhage with urgent treatment-requiring hydrocephalus support preterm patients with PHHC as a subgroup of shunttreated patients with aggravated risk not only of early but also of repeated shunt complications in the long-term perspective. Efforts in future studies should focus especially on pediatric patients with IVH-related hydrocephalus in order to reduce the complication rate and consider surgical treatment in correlation with neurodevelopmental and quality-of-life evaluations. Enhanced interdisciplinary and multicenter collaboration could strengthen the already accepted advancements in perinatal ICU treatment, ${ }^{1}$ promote the effort to reduce shunt-related morbidity, or, in the best case, attempt to prevent progressive development of hydrocephalus after IVH in preterm neonates. The latter ambition is currently still being pursued by neuroendoscopic lavage treatment after $\mathrm{IVH},{ }^{20}$ and the results of the feasibility and safety study could be interpreted as a first signal of a promising future perspective.

\section{Limitations of the Study}

Our investigations are limited by the retrospective and single-institution character of data analysis, and hence refer to a relatively small patient cohort. Although the applied inclusion criteria allow acquisition of valid and objective surgical outcome data based on a defined follow-up interval completed by the entire patient cohort, potential selection bias cannot be ruled out. This underlines the need for a high-volume, prospective, homogeneous, longterm data acquisition for pediatric hydrocephalus in order to provide reliable outcome evaluation.

\section{Conclusions}

Posthemorrhagic hydrocephalus of premature neonates as a consequence of perinatal IVH constitutes the most vulnerable and challenging patient population for VPS treatment. Higher-order IVH and GA less than 28 weeks were correlated with unfavorable shunt-related surgical outcome in terms of repeated shunt revisions within a defined follow-up interval of 5 years after VPS insertion. Temporizing measures such as VADs represent a rational and safe first-line strategy for immature infants with IVH who are in inadequate clinical or diagnostically less conclusive condition to receive direct VPS implantation.

\section{References}

1. Alan N, Manjila S, Minich N, Bass N, Cohen AR, Walsh M, et al: Reduced ventricular shunt rate in very preterm infants with severe intraventricular hemorrhage: an institutional experience. J Neurosurg Pediatr 10:357-364, 2012

2. Badhiwala JH, Hong CJ, Nassiri F, Hong BY, Riva-Cambrin J, Kulkarni AV: Treatment of posthemorrhagic ventricular dilation in preterm infants: a systematic review and meta- analysis of outcomes and complications. J Neurosurg Pediatr 16:545-555, 2015

3. Ballabh P: Intraventricular hemorrhage in premature infants: mechanism of disease. Pediatr Res 67:1-8, 2010

4. Bir SC, Konar S, Maiti TK, Kalakoti P, Bollam P, Nanda A: Outcome of ventriculoperitoneal shunt and predictors of shunt revision in infants with posthemorrhagic hydrocephalus. Childs Nerv Syst 32:1405-1414, 2016

5. Bock HC, Kanzler M, Thomale UW, Ludwig HC: Implementing a digital real-time hydrocephalus and shunt registry to evaluate contemporary pattern of care and surgical outcome in pediatric hydrocephalus. Childs Nerv Syst 34:457464, 2018

6. Brouwer AJ, Brouwer MJ, Groenendaal F, Benders MJNL, Whitelaw A, de Vries LS: European perspective on the diagnosis and treatment of posthaemorrhagic ventricular dilatation. Arch Dis Child Fetal Neonatal Ed 97:F50-F55, 2012

7. Cherian S, Whitelaw A, Thoresen M, Love S: The pathogenesis of neonatal post-hemorrhagic hydrocephalus. Brain Pathol 14:305-311, 2004

8. Chittiboina P, Pasieka H, Sonig A, Bollam P, Notarianni C, Willis BK, et al: Posthemorrhagic hydrocephalus and shunts: what are the predictors of multiple revision surgeries? J Neurosurg Pediatr 11:37-42, 2013

9. Flannery AM, Mitchell L: Pediatric hydrocephalus: systematic literature review and evidence-based guidelines. Part 1: Introduction and methodology. J Neurosurg Pediatr 14 (Suppl 1):3-7, 2014

10. Gurtner P, Bass T, Gudeman SK, Penix JO, Philput CB, Schinco FP: Surgical management of posthemorrhagic hydrocephalus in 22 low-birth-weight infants. Childs Nerv Syst 8:198-202, 1992

11. Köksal V, Öktem S: Ventriculosubgaleal shunt procedure and its long-term outcomes in premature infants with post-hemorrhagic hydrocephalus. Childs Nerv Syst 26:1505-1515, 2010

12. Kulkarni AV, Riva-Cambrin J, Butler J, Browd SR, Drake JM, Holubkov R, et al: Outcomes of CSF shunting in children: comparison of Hydrocephalus Clinical Research Network cohort with historical controls: clinical article. J Neurosurg Pediatr 12:334-338, 2013

13. Lam HP, Heilman CB: Ventricular access device versus ventriculosubgaleal shunt in post hemorrhagic hydrocephalus associated with prematurity. J Matern Fetal Neonatal Med 22:1097-1101, 2009

14. Levy ML, Masri LS, McComb JG: Outcome for preterm infants with germinal matrix hemorrhage and progressive hydrocephalus. Neurosurgery 41:1111-1118, 1997

15. Mazzola CA, Choudhri AF, Auguste KI, Limbrick DDJ Jr, Rogido M, Mitchell L, et al: Pediatric hydrocephalus: systematic literature review and evidence-based guidelines. Part 2: Management of posthemorrhagic hydrocephalus in premature infants. J Neurosurg Pediatr 14 (Suppl 1):8-23, 2014

16. Paulsen AH, Lundar T, Lindegaard KF: Twenty-year outcome in young adults with childhood hydrocephalus: assessment of surgical outcome, work participation, and health-related quality of life. J Neurosurg Pediatr 6:527-535, 2010

17. Radic JAE, Vincer M, McNeely PD: Outcomes of intraventricular hemorrhage and posthemorrhagic hydrocephalus in a population-based cohort of very preterm infants born to residents of Nova Scotia from 1993 to 2010. J Neurosurg Pediatr 15:580-588, 2015

18. Riva-Cambrin J, Kestle JRW, Holubkov R, Butler J, Kulkarni $\mathrm{AV}$, Drake J, et al: Risk factors for shunt malfunction in pediatric hydrocephalus: a multicenter prospective cohort study. J Neurosurg Pediatr 17:382-390, 2016

19. Robinson S: Neonatal posthemorrhagic hydrocephalus from prematurity: pathophysiology and current treatment concepts. J Neurosurg Pediatr 9:242-258, 2012 
20. Schulz M, Bührer C, Pohl-Schickinger A, Haberl H, Thomale UW: Neuroendoscopic lavage for the treatment of intraventricular hemorrhage and hydrocephalus in neonates. J Neurosurg Pediatr 13:626-635, 2014

21. Stein SC, Guo W: Have we made progress in preventing shunt failure? A critical analysis. J Neurosurg Pediatr 1:40-47, 2008

22. Vinchon M, Rekate H, Kulkarni AV: Pediatric hydrocephalus outcomes: a review. Fluids Barriers CNS 9:18, 2012

23. Volpe JJ: Intraventricular hemorrhage in the premature infant-current concepts. Part I. Ann Neurol 25:3-11, 1989

24. Wellons JC III, Shannon CN, Holubkov R, Riva-Cambrin J, Kulkarni AV, Limbrick DD Jr, et al: Shunting outcomes in posthemorrhagic hydrocephalus: results of a Hydrocephalus Clinical Research Network prospective cohort study. J Neurosurg Pediatr 20:19-29, 2017

\section{Disclosures}

The authors report no conflict of interest concerning the materi- als or methods used in this study or the findings specified in this paper.

\section{Author Contributions}

Conception and design: Bock, Ludwig. Acquisition of data: all authors. Analysis and interpretation of data: Bock. Drafting the article: Bock. Critically revising the article: Bock, Ludwig. Reviewed submitted version of manuscript: Bock. Approved the final version of the manuscript on behalf of all authors: Bock. Statistical analysis: Bock. Administrative/technical/material support: all authors. Study supervision: Bock, Ludwig.

\section{Supplemental Information}

\section{Previous Presentations}

Parts of the paper were presented as oral presentations at the 134th Annual Meeting "Chirurgie 2017" of the German Society of Surgery (DGC) in Munich, Germany, in March 2017.

\section{Correspondence}

Hans Christoph Bock: Universitätsmedizin Göttingen, Germany. cbock@gmx.de. 